Rev. Adm. Saúde - Vol. 18, № 70, jan. - mar. 2018

http://dx.doi.org/10.23973/ras.70.93

ARTIGO ORIGINAL

\title{
Elaboração e implantação de procedimento de solicitação de medicamentos manipulados personalizados
}

Elaboration and implementation of procedures for requesting custom manipulated drugs

\section{Ana Carolina Reis Guimarães', Priscila Faria Franca Marostegam², Cleuber Esteves Chaves $^{3}$, Vanusa Barbosa Pinto ${ }^{4}$}

1. Farmacêutica. Aprimoranda da Divisão de Farmácia do Instituto Central do Hospital das Clínicas da Faculdade de Medicina da USP, São Paulo SP

2. Farmacêutica. Farmacêutica da Divisão de Farmácia do Instituto Central do Hospital das Clínicas da Faculdade de Medicina da USP, São Paulo SP

3. Farmacêutico-Bioquímico. Diretor Técnico da Divisão de Farmácia do Instituto Central do Hospital das Clínicas da Faculdade de Medicina da USP, São Paulo SP

4. Farmacêutica. Diretora Técnica da Divisão de Farmácia do Instituto Central do Hospital das Clínicas da Faculdade de Medicina da USP, São Paulo SP

\section{RESUMO}

Introdução: O intuito da farmacotécnica é contribuir com as demais áreas da farmácia hospitalar, para que esta atenda suas atividades de serviço clínico e auxilie o atendimento ao paciente hospitalizado. Para que o paciente possa usufruir de um serviço de qualidade é necessário um sistema gerencial que reconheça suas necessidades e estabeleça padrões para assegurar a satisfação e recuperação do mesmo. Objetivo: Sistematizar o procedimento de solicitação de medicamentos manipulados. Método: Trata-se de um estudo descritivo, realizado no Setor de Manipulação da Unidade de Farmacotécnica Hospitalar. Foi realizada uma reunião com farmacêuticos participantes para apresentação e validação da ferramenta. Posteriormente foi elaborado um procedimento operacional padrão (POP), fluxograma e formulário de solicitação de medicamento manipulado. O mesmo era preenchido pelos farmacêuticos da 
central de avaliação de prescrição (CAP) e da assistência farmacêutica à internação (AFI) e encaminhado ao setor de manipulação, onde era feita a análise da viabilidade de manipulação do medicamento. Após a avaliação, a resposta era registrada em uma planilha Microsoft Excel e compartilhada com o profissional solicitante. Ao final da pesquisa foi aplicado um questionário de satisfação para os farmacêuticos solicitantes. Resultados: No período de estudo houve um total de 26 solicitações de medicamentos manipulados, os quais foram requisitados em sua maioria pela $\mathrm{AFI}$, representando $58 \%$ (15) dos pedidos. A clínica com o maior número de requisições de medicamentos manipulados foi a UTI da Divisão de Clínica Neurológica com 19\% (5) das solicitações. A classe farmacoterapêutica mais solicitadas de acordo com a classificação a Anatomical Therapeutic Chemical (ATC) foi a de antibacterianos para uso sistêmico, representando 27\% (7) dos medicamentos solicitados. Dos 26 medicamentos solicitados $81 \%$ (21) foram manipulados. O prazo de entrega dos medicamentos variou de 0 a 3 dias, sendo $57 \%$ (12) solicitações manipuladas e atendidas no mesmo dia. A via de administração em que houve o maior número de solicitações foi a oral com $38 \%$ (8). Os farmacêuticos demonstraram sua opinião quanto a ferramenta utilizada, avaliando atributos presentes no questionário de satisfação. Obteve-se um retorno de $64 \%$ dos questionários de satisfação. Destaca-se positivamente a avaliação dos farmacêuticos relacionada aos quesitos presentes no questionário, em que $100 \%$ dos farmacêuticos disseram que a ferramenta proposta deve ser implantada na rotina. Conclusão: A análise dos dados deste trabalho permitiu concluir que a ferramenta proposta para solicitação de medicamentos manipulados é considerada satisfatória e atende às expectativas dos usuários, ainda que algumas alterações e ajustes se façam necessárias.

Palavras-chave: medicamento manipulado, otimização de processos, farmácia hospitalar, boas práticas de manipulação.

\section{ABSTRACT}

Introduction: The aim of the pharmacotechnics is to contribute to the other areas of the hospital pharmacy, so that it can attend to its clinical service activities and assist the hospitalized patient. In order for the patient to be able to enjoy a quality service, a management system, that recognizes their needs and establishes standards to ensure their satisfaction and recovery, is necessary. Objective: To systematize the process of requesting manipulated drugs. Method: This is a descriptive study carried out in the Manipulation Sector of the Hospital Pharmaceutical Unit. A meeting was held with participating pharmacists to present and validate the tool. Subsequently, a standard operating procedure, flowchart and request form for manipulated drug was elaborated. The same was filled by the pharmacists of the prescription evaluation center and the pharmaceutical assistance to hospitalization (AFI) and sent to the manipulation sector, where the analysis of the viability of drug manipulation was made. After the evaluation, the response was recorded on a Microsoft Office Excel worksheet and shared with the requesting professional. At the end of the research, a satisfaction questionnaire was applied to the requesting pharmacists. Results: In the study period, there were a total of 26 
requests for manipulated drugs, which were requested in the majority by AFI, representing 58\% (15) of the requests. The clinic with the largest number of requests for medicines handled was the ICU of the Division of Neurological Clinic with 19\% (5) of the requests. The pharmacotherapeutic class most requested according to the classification of the Anatomical Therapeutic Chemical (ATC) was that of antibacterials for systemic use, representing $27 \%$ (7) of the drugs requested. Of the 26 drugs requested $81 \%$ (21) were handled. The delivery period of the drugs varied from 0 to 3 days, with $57 \%$ (12) requests handled and answered the same day. The route of administration with the highest number of requests was oral with $38 \%$ (8). Pharmacists showed their opinion about the tool used, evaluating attributes present in the satisfaction questionnaire. We obtained a return of $64 \%$ of satisfaction questionnaires. The evaluation of pharmacists was positively related to the questions present in the questionnaire, in which $100 \%$ of pharmacists said that the proposed tool should be implanted in the routine. Conclusion: The analysis of the data of this work allowed to conclude that the tool proposed for requesting manipulated drugs is considered satisfactory and meets the expectations of users, although some changes and adjustments are necessary.

Keywords: drug compounding, otimization of procedures, hospital pharmacy, good manipulation practices.

\section{INTRODUÇÃO}

Os medicamentos são insumos fundamentais na saúde e responsáveis pelo desfecho positivo em muitas doenças e agravos à saúde, incluindo aumento de sobrevida e redução de sofrimento ${ }^{1}$.

Como elemento principal do sistema de saúde, o paciente deve ter acesso aos medicamentos mais adequados ao seu perfil fisiopatológico, os quais podem ser produzidos tanto por indústrias farmacêuticas, quanto pelas farmácias de manipulação. Diante deste contexto, cada vez mais os farmacêuticos são solicitados para solucionar as necessidades farmacoterapêuticas individuais dos pacientes. A possibilidade de personalizar a terapêutica específica para cada paciente constitui uma importante razão para prescrição e preparação de medicamentos manipulados. Dessa forma, os medicamentos manipulados possibilitam atender não só questões como idade, sexo, metabolismo, condições físicas e psicológicas e a doença do paciente, mas também as necessidades do médico prescritor, de acordo com a estratégia terapêutica desejada ${ }^{2,3}$.

Recentemente, a farmácia hospitalar progrediu de uma unidade de armazenamento e distribuição, para um centro de manipulação especializado, responsável pelo preparo de centenas de requisições clínicas, as quais, em muitos casos, são únicas e não atendidas pela indústria 4 . Sendo assim, o 
intuito da farmacotécnica é contribuir com as demais áreas da farmácia hospitalar, para que esta atenda suas atividades de serviço clínico e auxilie diretamente no atendimento ao paciente hospitalizado ou ambulatorial ${ }^{5}$.

O setor magistral tem passado por transformações que visam ao atendimento das diretrizes de qualidade relacionadas ao fármaco, à gestão do processo e ao sistema de garantia de qualidade 6 . No Brasil, a preparação de medicamentos manipulados deve obedecer às normas da Resolução da Diretoria Colegiada (RDC) $n^{\circ} 67$ de 8 de outubro de 2007, que dispõe sobre boas práticas de manipulação de preparações magistrais e oficinais para uso humano em farmácias. Esta determina que é atribuição da gerência superior estar comprometida com as atividades de boas práticas farmacêuticas, garantindo a melhoria contínua e a garantia da qualidade. Além disso, o farmacêutico deve desenvolver e atualizar regularmente as diretrizes e procedimentos relativos aos aspectos operacionais da manipulação ${ }^{7}$.

Para que o paciente possa usufruir de um serviço de qualidade é necessário um sistema gerencial que reconheça suas necessidades, estabeleça padrões e os mantenha para assegurar a satisfação e recuperação do mesmo ${ }^{8}$.

Uma maneira de iniciar a padronização se dá por meio da compreensão de como ocorre todo o processo. Os padrões são definidos visando o estabelecimento das diretrizes para o controle e melhoria contínua da qualidade, que irão impulsionar as organizações para o desenvolvimento da melhoria de seus processos e resultados ${ }^{9,10}$.

\section{JUSTIFICATIVA}

Esse trabalho justifica-se pelo fato que não há um procedimento estruturado e sistematizado que apresente as etapas, documentos e informações necessárias para solicitação, avaliação e viabilidade da manipulação do medicamento. O desenvolvimento do procedimento deverá permitir aos envolvidos uma melhor compreensão e otimização do processo.

\section{OBJETIVOS}

Desenvolver e implantar um procedimento para a solicitação, avaliação e resposta da viabilidade de manipulação de medicamentos manipulados personalizados.

Objetivos específicos:

- Elaborar procedimento operacional padrão (POP) e fluxograma da rotina de solicitação e avaliação de medicamentos manipulados;

- Elaborar formulário de solicitação de medicamentos manipulados;

- Aplicar procedimento; 
- Avaliar quantitativamente as solicitações de medicamentos manipulados: número de medicamentos solicitados e número de dias para a entrega dos medicamentos;

- Avaliar qualitativamente os medicamentos solicitados: clínica que houve maior demanda de solicitações, classificação farmacoterapêutica e vias de administração;

- Avaliar quais medicamentos não foram viáveis de manipular;

- Avaliar a satisfação dos solicitantes quanto ao procedimento estabelecido.

\section{MATERIAL E MÉTODOS}

\section{Local e tipo de estudo}

O estudo descritivo foi realizado no Setor de Manipulação da Unidade de Farmacotécnica Hospitalar (UFAR), localizado no Prédio dos Ambulatórios do Hospital das Clínicas da Faculdade de Medicina da Universidade de São Paulo (PAMB FMUSP).

\section{Período de estudo}

O período de coleta de dados compreendeu de 20 de setembro de 2017 a 10 de dezembro de 2017.

\section{Critérios de inclusão}

Solicitações de medicamentos manipulados personalizados para pacientes internados; consultas feitas por farmacêuticos da Central de Avaliação de Prescrição (CAP) que fazem avaliação da prescrição e da Assistência Farmacêutica à Internação (AFI) que realizam o atendimento das prescrições.

\section{Critérios de exclusão}

Solicitações de medicamentos manipulados para uso ambulatorial.

\section{Elaboração do procedimento operacional padrão (POP)}


Para elaboração do procedimento, foi realizada uma reunião com os farmacêuticos da AFI e CAP para discussão, identificação das necessidades da equipe farmacêutica e explicação do projeto.

Foi utilizado o modelo institucional de POP para registrar as etapas a serem executadas do procedimento.

Como representação gráfica do procedimento estabelecido, foi empregada a ferramenta da qualidade fluxograma.

Para elaboração do formulário, foram consideradas as necessidades identificadas na reunião com os farmacêuticos, as orientações da RDC 67/2007 e as informações técnicas necessárias para a manipulação dos medicamentos. Foi utilizado o instrumento Formulários Google para a coleta das solicitações de medicamentos manipulados.

\section{Aplicação do procedimento proposto}

Os farmacêuticos foram treinados na nova rotina, preenchiam o formulário e 0 encaminhava para o setor de manipulação. O farmacêutico da manipulação fazia a avaliação farmacotécnica e viabilidade da manipulação, considerando:

- $\quad$ indicação terapêutica;

- $\quad$ referências bibliográficas;

- $\quad$ avaliação farmacêutica da prescrição;

- disponibilidade de matéria-prima e insumos e

- $\quad$ capacidade de manipulação.

Quando os farmacêuticos da AFI e CAP preenchiam o formulário um alerta de aviso era recebido por e-mail ou os farmacêuticos da manipulação acessavam diretamente o Formulário Google disponível no Google Drive.

Os dados das solicitações foram registrados no Formulário Google, conforme variáveis abaixo:

- registro geral do Hospital das Clínicas (RGHC);

- nome do paciente;

- medicamento;

- concentração;

- posologia; 
- via de administração;

- duração do tratamento;

- clínica;

- leito;

- nome do prescritor;

- unidade solicitante;

- farmacêutico solicitante;

- data da solicitação;

- horário da solicitação;

- viabilidade da manipulação;

- justificativa;

- data da resposta;

- horário e

- data da entrega.

\section{Avaliação quantitativa das solicitações de medicamentos manipulados}

Através dos dados registrados na planilha Microsoft Excel, extraída do Formulário Google, foram quantificados o número total de solicitações de medicamentos e o número de dias necessários para a entrega das manipulações.

\section{Avaliação qualitativa das solicitações de medicamentos manipulados}

Utilizaram-se os dados registrados na planilha Microsoft Excel, extraída do Formulário Google. Foi feita a análise da clínica com maior número de solicitações de medicamentos manipulados e as vias de administração de medicamentos mais requisitados. Os medicamentos solicitados foram classificados de acordo com a Anatomical Therapeutic Chemical (ATC).

\section{Avaliação da não viabilidade de manipulação dos medicamentos solicitados}


No Formulário de Solicitação de Medicamento Manipulado, havia um quesito referente a justificativa da não viabilidade de manipulação do medicamento solicitado, o qual os farmacêuticos do setor preenchiam quando não era viável a manipulação do produto. Por meio destes registros, foi possível analisar o motivo pelo qual não foi realizada a manipulação do medicamento.

\section{Avaliação da satisfação dos solicitantes}

A satisfação dos solicitantes foi avaliada por meio de Formulários Google, no qual foram analisadas as perguntas:

- "você utilizou a ferramenta?";

- "facilidade de acesso";

- "facilidade para preencher o formulário";

- “as perguntas são claras e objetivas?";

- "tempo de resposta da viabilidade ou não da manipulação e prazo de atendimento";

- "a ferramenta facilitou a comunicação com a manipulação?";

- "a ferramenta sistematizou a solicitação de medicamentos manipulados?";

- "o novo processo de solicitação de medicamentos manipulados deve continuar e ser implantado na rotina?" e

- "considerações/sugestões".

As sugestões registradas em campo aberto foram agrupadas por semelhança e tabuladas.

O questionário foi aplicado ao final da coleta de dados para os farmacêuticos.

\section{Desenho do estudo}

O estudo será realizado conforme fluxograma da Figura 1. 


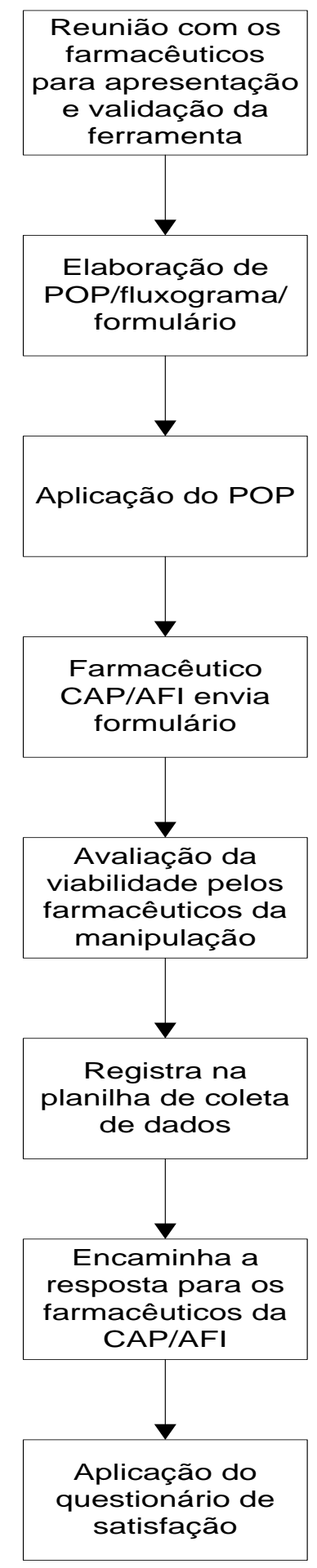

Figura 1. Fluxograma das etapas da pesquisa.

\section{Análise estatística}

Os dados coletados foram tabulados em planilha Microsoft Excel 2007 e analisados por meio de análise estatística descritiva, calculando frequência e porcentagem. 


\section{Aspectos éticos}

O projeto de pesquisa foi submetido à avaliação do Comitê de Ética em Pesquisa e da Comissão de Ética para Análise de Projetos de Pesquisa do HCFMUSP (CAPPesq).

Todos os farmacêuticos que preencheram os critérios de inclusão receberam o Termo de Consentimento Livre e Esclarecido (TCLE), que foi lido e discutido junto ao pesquisador responsável antes do início da pesquisa. Nesse momento, os farmacêuticos foram orientados sobre o direito de retirar o consentimento a qualquer momento, sem prejuízo. Todos os farmacêuticos assinaram o documento.

Os questionários respondidos foram mantidos em banco de dados eletrônico de propriedade do pesquisador responsável. A identidade dos participantes foi preservada.

\section{RESULTADOS E DISCUSSÃO}

Foram elaborados POP e fluxograma para padronizar a rotina proposta, já que a utilização de padronização nos processos faz com que se tenha o cumprimento adequado das atividades realizadas, de modo que cada pessoa tenha condição de assumir a responsabilidade pelos resultados do próprio trabalho ${ }^{11}$. O POP, assim como o fluxograma, foi escrito de forma detalhada para obtenção de uniformidade da rotina operacional permitindo a verificação de cada uma das suas etapas. Ambos estão disponibilizados no sistema informatizado da instituição para ser consultado sempre que necessário.

Foi desenvolvido um formulário online de solicitação de medicamento manipulado no Formulário Google disponibilizado no Google Drive conforme mostrado na Figura 2. Para explicar como deveria ser feito o preenchimento do formulário, foi realizada reunião com os farmacêuticos participantes. A ferramenta Formulários Google apresenta as respostas agrupadas, algumas em forma de gráfico, facilitando a análise. Além disso, gera uma planilha no formato Microsoft Excel com todas as respostas, tornando fácil a extração e comparação de informações obtidas por meio de diferentes perguntas. Quanto às questões que exigiam respostas discursivas, foi realizada análise qualitativa dos seus conteúdos e agrupamento por semelhança. 


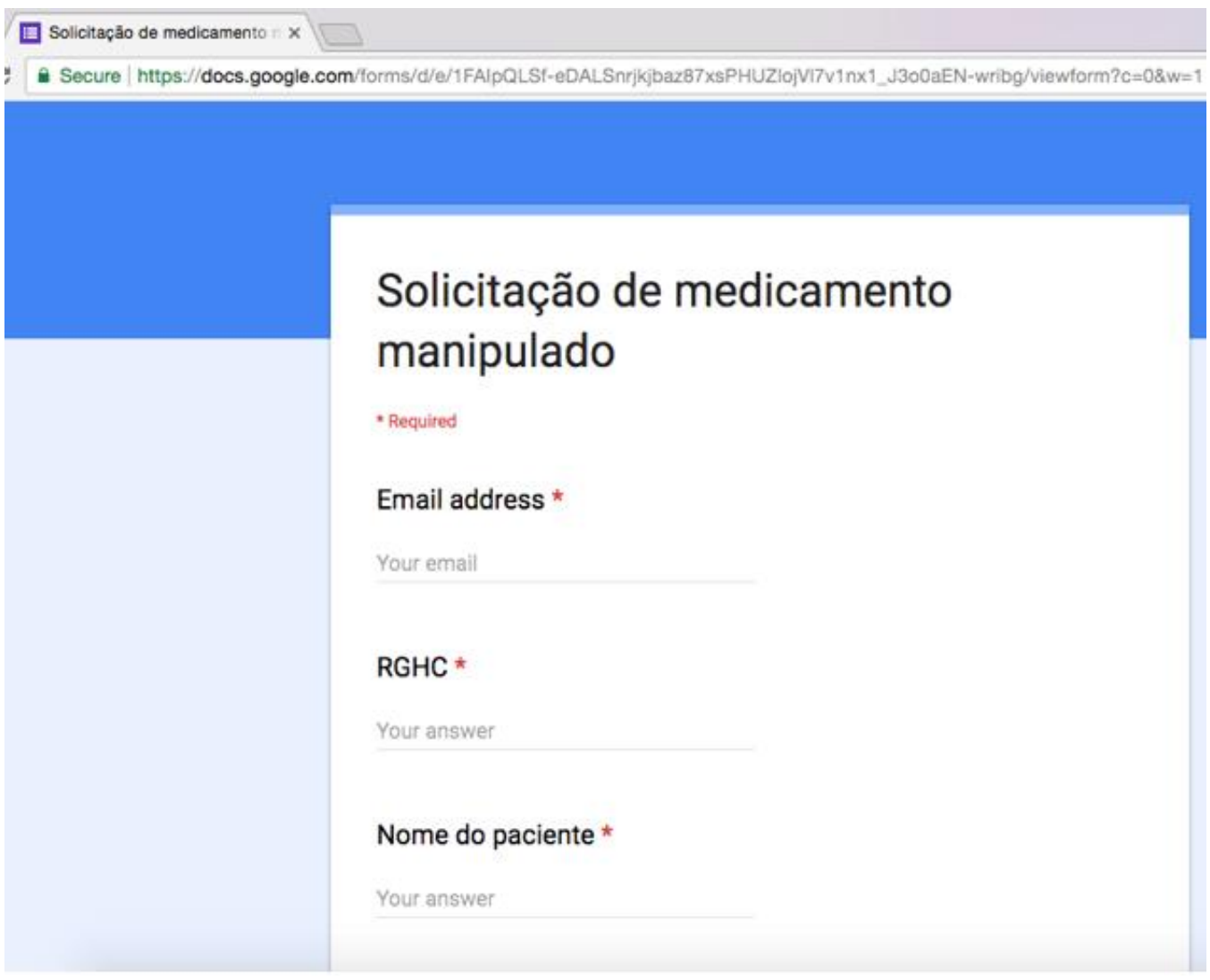

Figura 2. Formulário de solicitação de medicamento manipulado disponível no Formulários Google

No período de 20 de setembro de 2017 a 10 de dezembro de 2017 foram solicitados 26 medicamentos, conforme demonstrado na Tabela 1.

Tabela 1. Quantidade de medicamentos solicitados, no ICHC no período de 20 de setembro a 10 de dezembro de 2017.

\begin{tabular}{lrrr}
\hline $\begin{array}{l}\text { Meses de } \\
2017\end{array}$ & № de solicitações & Dias com solicitação & № de solicitações/dia \\
\hline Setembro & 5 & 4 & 1,3 \\
Outubro & 7 & 4 & 1,8 \\
Novembro & 11 & 8 & 1,4 \\
Dezembro & 3 & 2 & 1,5 \\
\hline Total geral & 26 & 18 & 1,4 \\
Média mês & 6,5 & 4,5 & - \\
\hline
\end{tabular}

A unidade que mais solicitou foi a de Assistência Farmacêutica à Internação (AFI), representando $15(58 \%)$ dos pedidos de medicamentos manipulados 
(Figura 3). Os demais medicamentos foram solicitados pela CAP 11 (42\%) de acordo com a Figura 3. Tal porcentagem pode ser justificada pela maior parte das clínicas serem atendidas pela AFI, cerca de 435 leitos. A avaliação farmacêutica identifica potenciais problemas relacionados a medicamentos e previne grande parte deles ${ }^{12}$. Como o farmacêutico nesse processo analisa a posologia, via de administração e indicação terapêutica, tal processo pode resultar em intervenções, como por exemplo, solicitação de adequação de forma farmacêutica para o setor de manipulação, otimizando a terapia medicamentosa do paciente de acordo com suas necessidades.

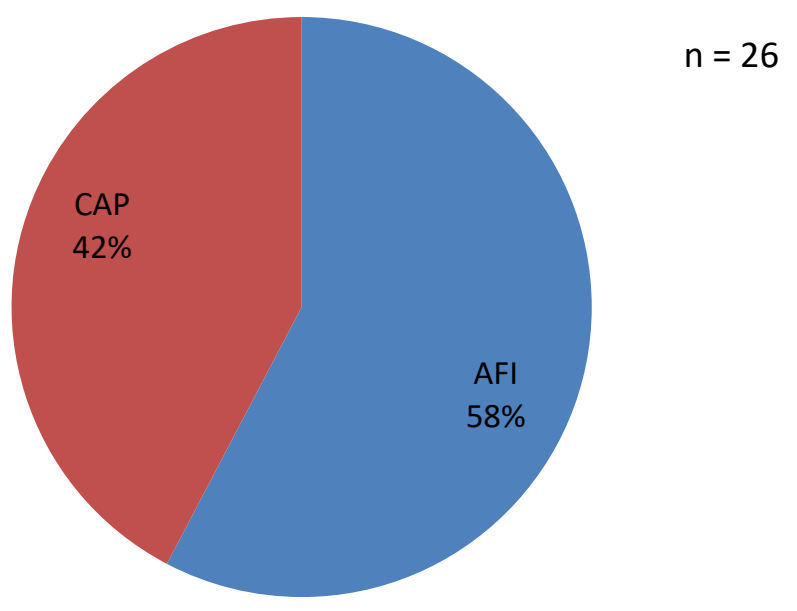

Figura 3. Distribuição dos medicamentos solicitados por unidade solicitante no ICHC, no período de 20 de setembro a 10 de dezembro de 2017.

A clínica que mais solicitou medicamentos manipulados foi a UTI da Divisão de Clínica Neurológica (Figura 4), o que pode ser explicado pela presença de farmacêutico clínico nas visitas multiprofissionais do setor, visto que esse profissional é o elo entre a prescrição e administração dos medicamentos, o que contribui significativamente para a melhoria da farmacoterapia. A presença do farmacêutico aumenta o número de intervenções realizadas, favorece o uso racional de medicamentos, seleciona o fármaco e/ou forma farmacêutica com menor probabilidade de causar complicações, aumenta a segurança do paciente e contribui para a redução de custos associados à prescrição médica ${ }^{13}$. 

Enfermaria de Geriatria

UTI Emergência PS Cirurgia

Enfermaria Geral de Pronto Socorro

Enfermaria da Divisão de Clínica Urológica

UTI 9o da Divisão de Clínica Cirúrgica II

Enfermaria Transplante Renal

Enfermaria PS Cirurgia Emergência

Enfermaria Cuidados Paliativos

Enfermaria da Divisão de Clínica de...

UTI Clinica de Moléstias Infecciosas e Parasitárias

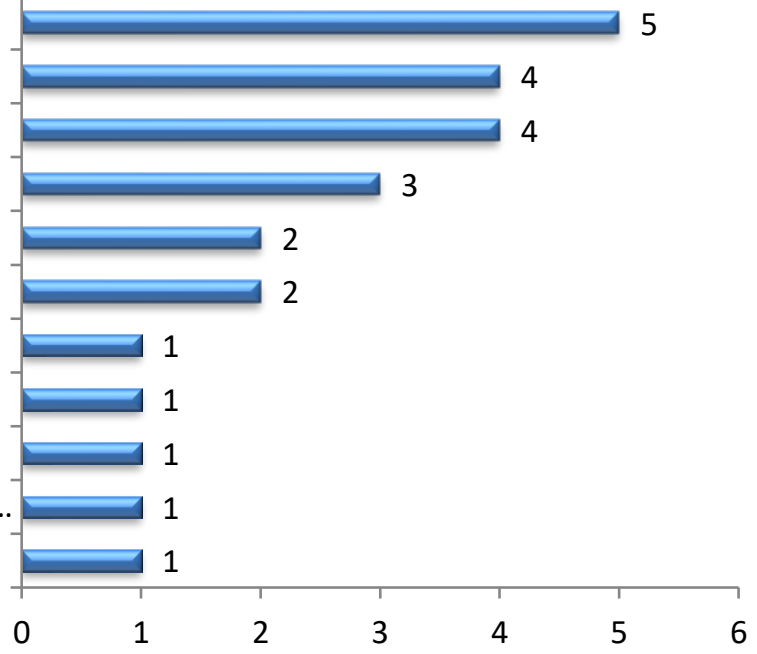

Figura 4. Distribuição das solicitações de medicamentos manipulados por clínica no ICHC, no período de 20 de setembro a 10 de dezembro de 2017.

Segundo a classificação Anatomical Therapeutic Chemical (ATC), as classes farmacoterapêuticas mais solicitadas foram: antibacterianos para uso sistêmico, representando 7 (27\%), medicamentos para tratamento da tuberculose 5 (19\%), corticosteróides para uso sistêmico 4 (15\%), preparações antianêmicas $3(11 \%)$, antivíricos para uso sistêmico $2(8 \%)$, analgésicos 1 (4\%), antimicóticos para uso sistêmico 1(4\%), vasoprotetores $1(4 \%)$, preparações estomatológicas 1 (4\%) e antipsoriáticos 1 (4\%), conforme Figura 5. 


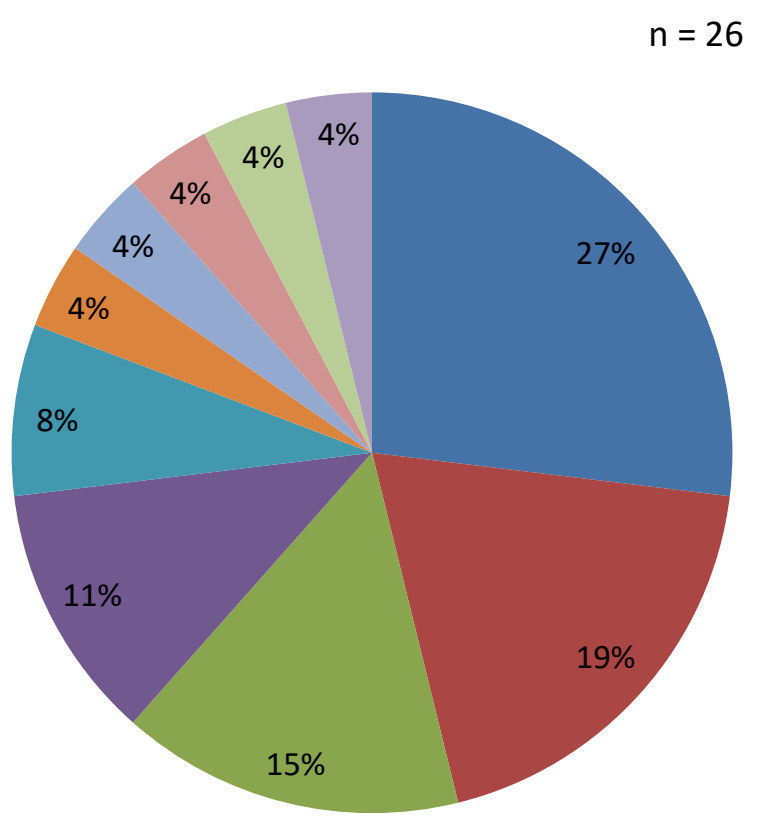

Antibacterianos para uso
sistêmico
Medicamentos para
tratamento da tuberculose
Corticosteróides para uso
sistêmico
Preparações antianêmicas
Antivíricos para uso sistêmico
Análgésicos
Antimicóticos para uso
sistêmico
Vasoprotetores

Figura 5. Distribuição das classes farmacoterapêuticas de medicamentos manipulados solicitados no ICHC, no período de 20 de setembro a 10 de dezembro de 2017.

Dos 26 medicamentos solicitados para o setor de manipulação, 21 (81\%) foram manipulados e 5 (19\%) não foram manipulados, conforme demonstrado a seguir na tabela 2 e figura 6 :

Tabela 2. Viabilidade de manipulação dos medicamentos solicitados por mês, no ICHC, no período de 20 de setembro a 10 de dezembro de 2017.

\begin{tabular}{lrrr}
\hline Meses & \multicolumn{2}{c}{ Medicamentos manipulados } & \begin{tabular}{r} 
Total de \\
\cline { 2 - 3 }
\end{tabular} \\
\cline { 2 - 3 } Não (\%) & Sim (\%) & medicamentos \\
\hline Setembro & $3(60 \%)$ & $2(40 \%)$ & 5 \\
Outubro & $0(0 \%)$ & $7(100 \%)$ & 7 \\
Novembro & $0(0 \%)$ & $11(100 \%)$ & 11 \\
Dezembro & $2(67 \%)$ & $1(33 \%)$ & 3 \\
\hline Total geral & 5 & 21 & 26 \\
\hline
\end{tabular}




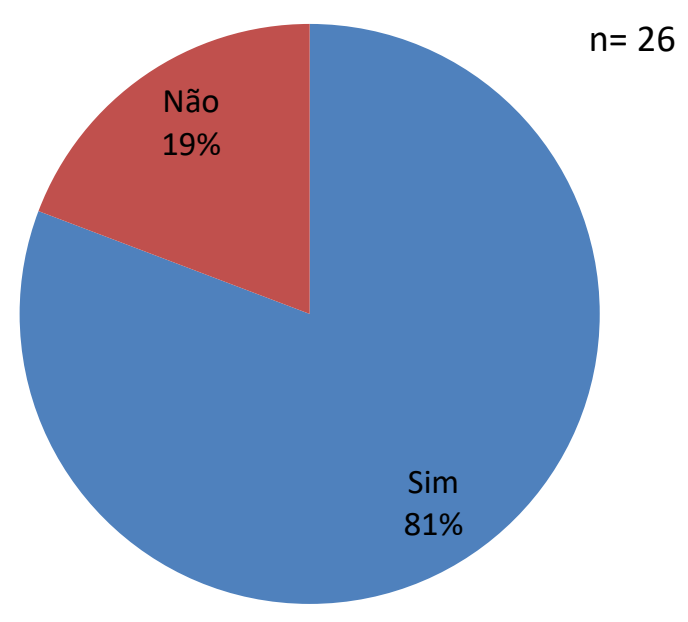

Figura 6. Viabilidade de manipulação dos medicamentos solicitados no ICHC, no período de 20 de setembro a 10 de dezembro de 2017.

O prazo de entrega dos medicamentos variou de 0 a 3 dias, sendo a maior parte manipulada e atendida no mesmo dia $12(57 \%)$, como mostrado na figura 7. As manipulações entregues após 1 dia da solicitação foram devido ao pedido ter sido feito tardiamente, após horário de expediente, ou por razões logísticas que inviabilizavam a manipulação do medicamento naquele momento.

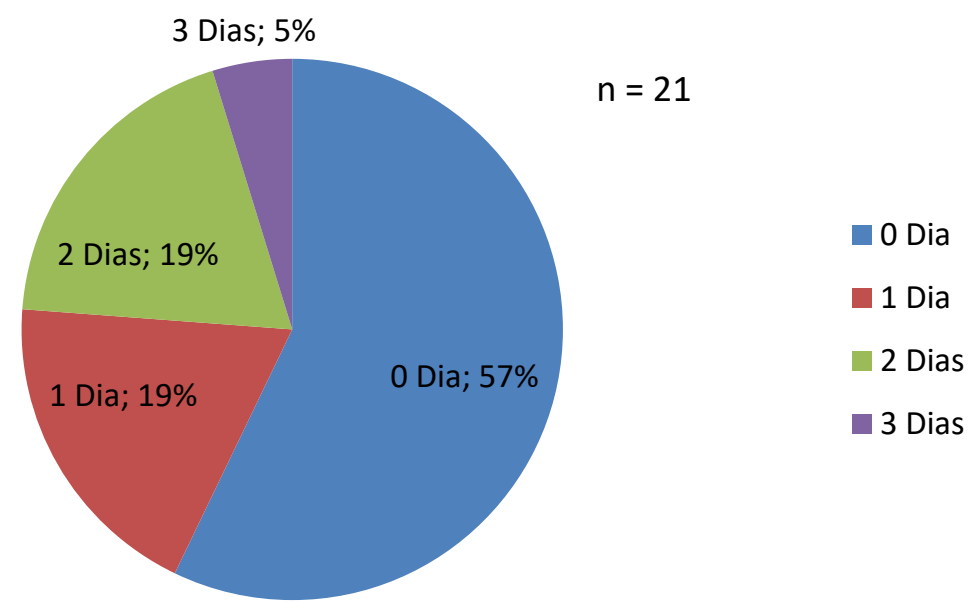

Figura 7. Prazo de entrega dos medicamentos manipulados solicitados no ICHC, no período de 20 de setembro a 10 de dezembro de 2017. 
A via de administração em que houve o maior número de solicitações de medicamentos viáveis de manipular foi a oral $8(38 \%)$, seguida da sonda nasoentérica $5(24 \%)$ e via retal $5(24 \%)$, conforme demonstrado na Figura 8 . 0 maior número de solicitações nessas vias, pode ser justificado por serem seguras e com menor prevalência de complicações, resultando em maior comodidade ao paciente e de administração pela equipe de enfermagem.

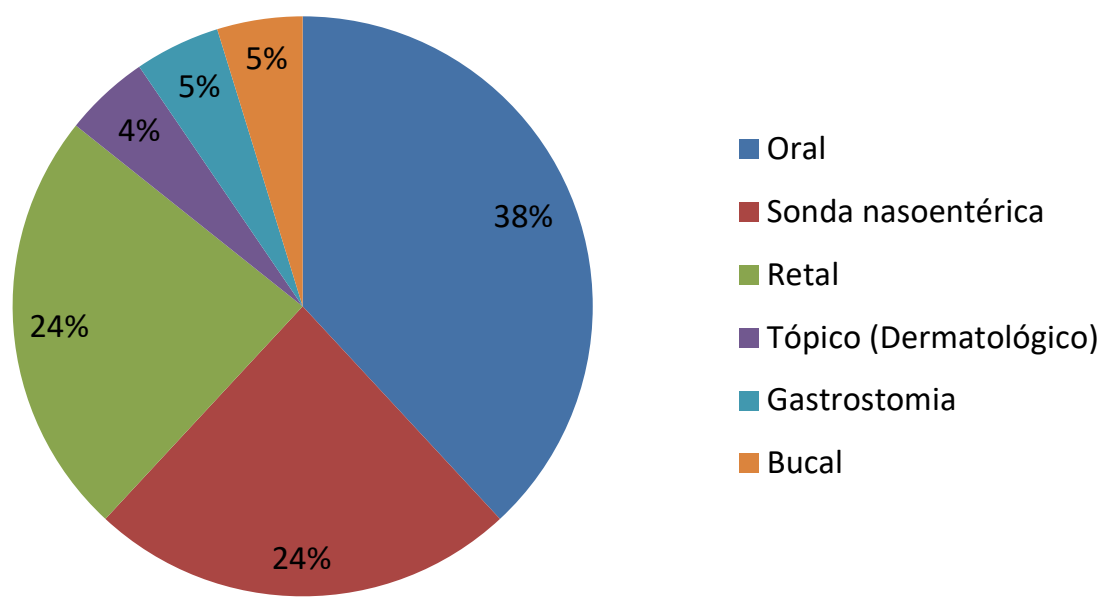

Figura 8. Vias de administração dos medicamentos manipulados no ICHC, no período de 20 de setembro a 10 de dezembro de 2017.

Uma dificuldade encontrada com esta informação na ferramenta e rotina proposta de solicitação de medicamentos manipulados foi que em um primeiro momento a via de administração dos medicamentos foi criada como campo aberto, o que permitiu que cada farmacêutico preenchesse a via de forma não padronizada. Como melhoria, a via de administração será um campo de resposta fechada e padronizada de acordo com as vias de administração utilizadas nos sistemas informatizados do HCFMUSP, que seguiu a terminologia padronizada pela Agência Nacional de Vigilância Sanitária (Anvisa) no vocabulário controlado de formas farmacêuticas, vias de administração e embalagens de medicamentos.

A Tabela 3 apresenta os medicamentos manipulados solicitados que não foram manipulados.

Tabela 3. Medicamentos solicitados não manipulados, de acordo com a via de administração no ICHC, no período de 20 de setembro a 10 de dezembro de 2017. 


\begin{tabular}{|c|c|c|}
\hline Medicamento & Via de administração & Motivo \\
\hline $\begin{array}{l}\text { Fludrocortisona } 50 \mathrm{mcg} \\
\text { (suspensão oral) }\end{array}$ & Gastrostomia & $\begin{array}{l}\text { O medicamento já havia } \\
\text { sido manipulado para } \\
\text { tratamento completo. }\end{array}$ \\
\hline $\begin{array}{l}\text { Efavirenz } 600 \mathrm{mg} \\
\text { (suspensão oral) }\end{array}$ & Sonda nasoentérica & $\begin{array}{l}\text { Não foi encontrado na } \\
\text { literatura sobre a } \\
\text { estabilidade do mesmo } \\
\text { após ser triturado. }\end{array}$ \\
\hline $\begin{array}{l}\text { Abacavir } 300 \mathrm{mg} \\
\text { (suspensão oral) }\end{array}$ & Sonda nasoentérica & $\begin{array}{l}\text { Não foi encontrado na } \\
\text { literatura sobre a } \\
\text { estabilidade do mesmo } \\
\text { após ser triturado. }\end{array}$ \\
\hline $\begin{array}{l}\text { Rifampicina } 600 \mathrm{mg}+\text { Isoniazida } \\
300 \mathrm{mg}+\text { Pirazinamida } 1600 \mathrm{mg} \\
\text { + Etambutol } 1100 \mathrm{mg} \\
\text { (suspensão oral) }\end{array}$ & Sonda nasoentérica & $\begin{array}{l}\text { A formulação está em fase } \\
\text { de desenvolvimento. }\end{array}$ \\
\hline $\begin{array}{l}\text { Fludrocortisona } 50 \mathrm{mcg} \\
\text { (suspensão oral) }\end{array}$ & Sonda nasoentérica & $\begin{array}{l}\text { O medicamento já havia } \\
\text { sido manipulado para } \\
\text { tratamento completo. }\end{array}$ \\
\hline
\end{tabular}

Os medicamentos fludrocortisona $50 \mathrm{mcg}$ na forma farmacêutica suspensão oral via gastrostomia (GTRS) e via sonda nasoentérica (SNE), já haviam sido produzidos previamente em quantidade suficiente para atender o tratamento completo dos pacientes. No caso do efavirenz $600 \mathrm{mg}$ e abacavir $300 \mathrm{mg}$ ambos na forma farmacêutica suspensão, não foram encontradas na literatura informações sobre a estabilidade dos mesmos após serem triturados. $\mathrm{O}$ medicamento Rifampicina $600 \mathrm{mg}$ + Isoniazida $300 \mathrm{mg}$ + Pirazinamida 1600 $\mathrm{mg}+$ Etambutol $1100 \mathrm{mg}$ na forma de suspensão oral não foi manipulado, pois a formulação está em fase de desenvolvimento.

Um dos instrumentos indispensáveis para o bom desempenho da ação programática é a avaliação contínua de todo processo e dos seus resultados. De acordo com tal conceito, a pesquisa de satisfação do cliente tem sido uma ferramenta importante para verificar e mensurar os procedimentos, produtos e serviços oferecidos pelas instituições ${ }^{14}$.

Ao final do período de coleta de dados, foi enviado um questionário de pesquisa de satisfação sobre o Formulário de Solicitação de Medicamentos Manipulados, por meio do Formulários Google, para um total de 14 farmacêuticos da AFI e CAP. Obteve-se um retorno de $9(64 \%)$ questionários de satisfação respondidos (Figura 9). A ausência de resposta de 5 (36\%) questionários é justificada pela não utilização da ferramenta pelos mesmos. 


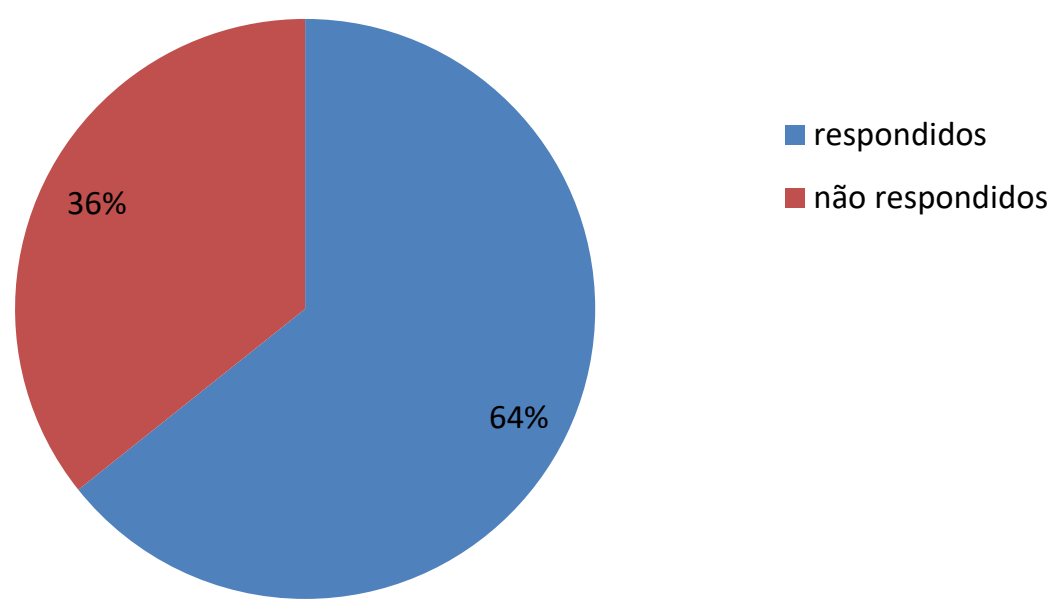

Figura 9. Questionários da pesquisa de satisfação respondidos no ICHC, no período de 20 de setembro a 10 de dezembro de 2017.

Os farmacêuticos demonstraram sua opinião quanto a ferramenta utilizada, avaliando atributos presentes no questionário. Em relação às facilidades de acesso e para preencher o formulário, $6(66,7 \%)$ avaliaram como "ótimo" e 3 $(33,3 \%)$ como "bom", o que mostra que o instrumento facilitou a rotina de solicitação de medicamentos manipulados (Figura 10). 


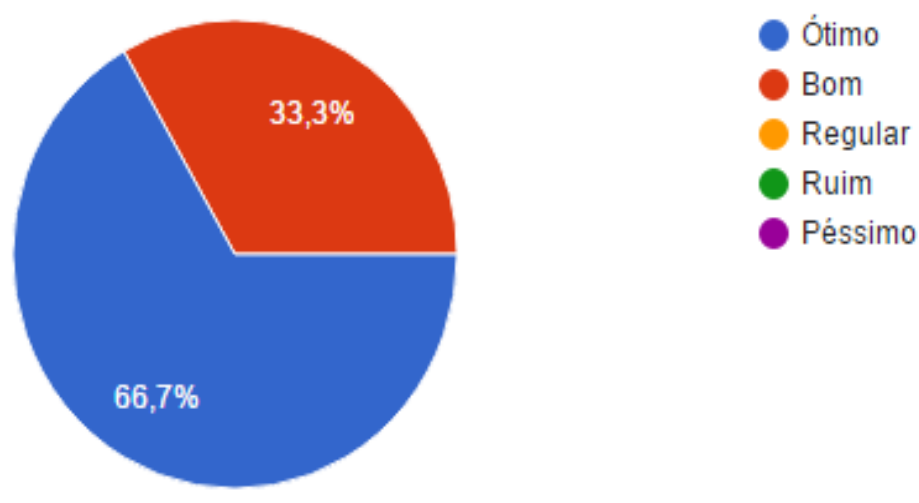

Figura 10. Facilidades de acesso e preenchimento do formulário no $\mathrm{ICHC}$, no período de 20 de setembro a 10 de dezembro de 2017.

No que se refere a objetividade e clareza das perguntas utilizadas no Formulário de Solicitação de Medicamento Manipulado, 7 (77,8\%) dos farmacêuticos classificaram como "ótimo" e 2 (22,2\%) como "bom", conforme mostrado na Figura 11.
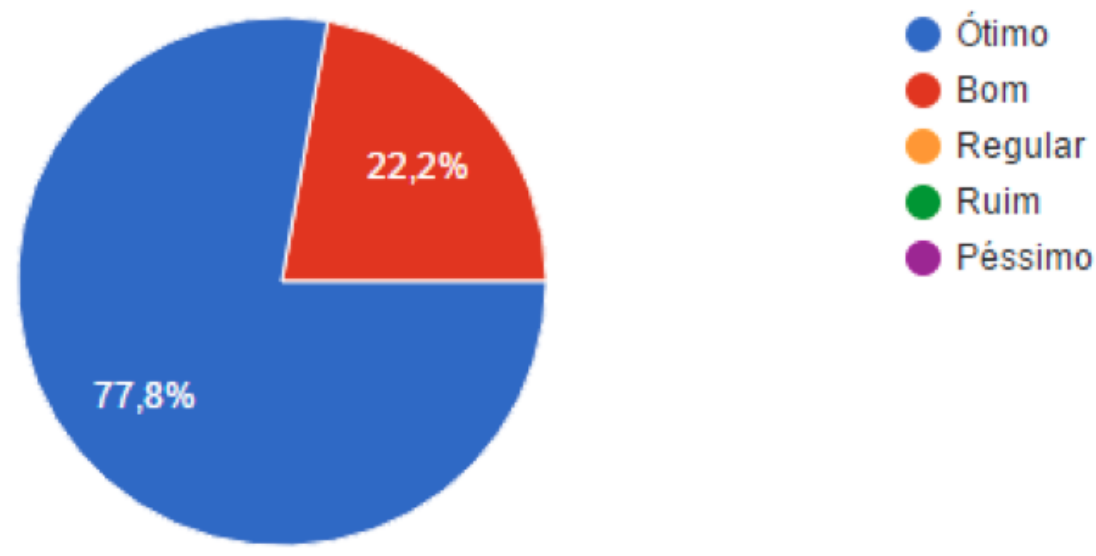

Figura 11. Objetividade e clareza das perguntas utilizadas no formulário no ICHC, no período de 20 de setembro a 10 de dezembro de 2017.

Os quesitos referentes a "relação ao tempo de resposta da viabilidade ou não da manipulação e prazo de atendimento das solicitações" e "clareza e objetividade das respostas da manipulação em relação as solicitações de 
medicamentos manipulados" foram aqueles em que os farmacêuticos se mostraram menos satisfeitos, $1(11,1 \%)$ responderam "regular". Ainda assim obteve-se um índice de 5 (55,6\%) classificando como "ótimo" e 3 (33,3\%) como "bom" (Figura 12).
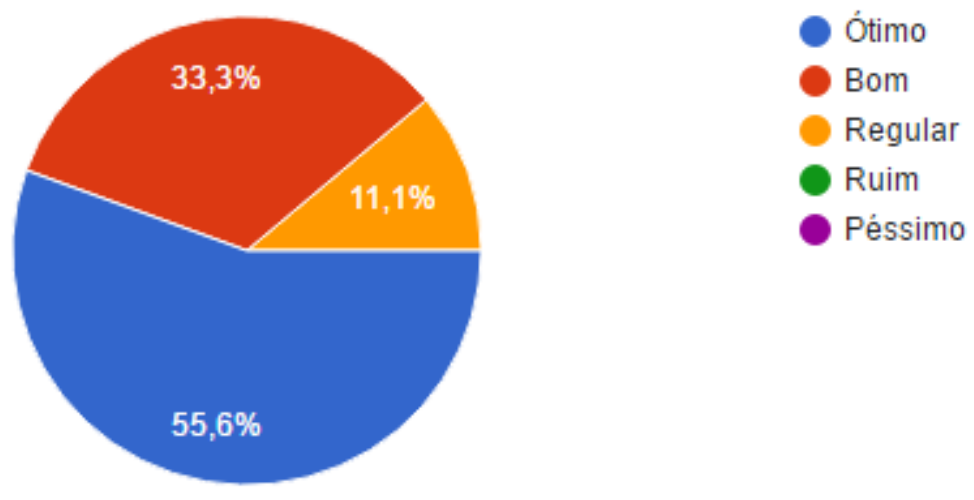

$$
\mathrm{n}=9
$$

Figura 12. Relação ao tempo de resposta da viabilidade ou não da manipulação, prazo de atendimento das solicitações e clareza e objetividade das respostas da manipulação no ICHC, no período de 20 de setembro a 10 de dezembro de 2017.

Destaca-se positivamente a avaliação dos farmacêuticos relacionada aos quesitos "a ferramenta facilitou a comunicação com a manipulação?", "a ferramenta sistematizou a solicitação de medicamentos manipulados?" e "o novo processo de solicitação de medicamentos manipulados deve continuar e ser implantado na rotina?", em que $9(100 \%)$ dos farmacêuticos responderam "sim".

No campo "considerações/sugestões", foram deixadas algumas propostas de melhorias, na maioria relacionadas a inclusão de informações, que serão consideradas e implantadas no formulário.

Para Kotler $(1998)^{15}$, o conceito de satisfação é o sentimento de prazer ou desapontamento resultante da comparação do desempenho esperado pelo produto ou resultado em relação às expectativas das pessoas. Para analisar a satisfação do cliente, as organizações precisam entender que as expectativas de cada consumidor são diferentes umas das outras. Dessa forma, é necessário conhecer os seus clientes e compreender que eles podem estar (ou não) satisfeitos no momento em que preenchem o questionário da pesquisa. Assim, é preciso que a aplicação da pesquisa seja feita de forma periódica, como por exemplo, anualmente, uma vez que a opinião do cliente não é definitiva nem estática. 
O formulário foi considerado adequado pelos farmacêuticos, como pôde ser constatado pelo retorno registrado no campo referente a

"considerações/sugestões" do formulário de pesquisa de satisfação e pelo índice de $64 \%$ de retorno alcançado. Estudos anteriores, nos quais utilizou-se 0 questionário como técnica de coleta, mostram que o índice de retorno, em média, é de $30 \%$ nas pesquisas de satisfação de clientes $^{16}$.

Embora a avaliação geral da pesquisa tenha sido considerada positiva, 0 resultado relativo ao cumprimento de prazos mostrou-se como uma oportunidade de melhoria para o setor de manipulação. É necessário enfatizar que cada medicamento manipulado possui particularidades técnicas e que às vezes demandam um tempo maior para a sua produção, respeitando suas especificidades. Nem sempre os profissionais têm conhecimento das técnicas utilizadas na manipulação e não compreendem o tempo necessário para realização das mesmas. Vale ressaltar que em alguns casos não existem formulações do medicamento solicitado na literatura, sendo necessário efetuar pesquisas e desenvolver a formulação.

\section{CONCLUSÃO}

Nos dias de hoje, a aposta na eficiência e excelência da gestão vem se destacando como grande diferencial nas organizações e, dessa forma, é preciso inovar e implantar continuamente boas práticas de gestão para ter sucesso e gerar melhores resultados.

A busca do nível de satisfação em relação aos serviços oferecidos, ou seja, mensurar o quanto as expectativas dos usuários estão sendo atendidas, oportuniza aos gestores direcionar melhorias a partir da visão daqueles que utilizam os serviços.

O uso da ferramenta Formulários Google foi importante e demonstrou ser ágil, interativo e sem a necessidade de haver gasto com material impresso.

A análise dos dados deste trabalho permitiu concluir que a ferramenta proposta para solicitação de medicamentos manipulados é considerada satisfatória e atendem às expectativas dos usuários, ainda que algumas alterações e ajustes se façam necessárias.

\section{REFERÊNCIAS}

1 Luiza VL, Tavares NUL, Oliveira MA, Arrais PSD, Ramos LR, da Silva Dal Pizzol LR, et al. Gasto catastrófico com medicamentos no Brasil. Rev Saude Publica. 2016;50 (supl 2):15s.

5. Barbosa CM, Pinto S. Medicamentos manipulados: Que perspectivas? Farmácia Portuguesa, 2000:123. 54-60. 
6. Barbosa CM. Os medicamentos manipulados em Portugal. Rev ANFARMAG. 2006: 59: 42- 46.

7. Nascimento EP, Ventura DM, Lima FA, Pereira CR. Estudo fármacoeconômico do perfil de consumo de medicamentos produzidos pelo Setor de Farmacotécnica do Serviço de Farmácia do Hospital Geral de Bonsucesso. Rev. Bras. Farm., 88(2): 74-76, 2007.

8. Gomes MJVM. Ciências Farmacêuticas: uma abordagem em farmácia hospitalar, 1ํㅡㄹ ed. São Paulo: Editora Atheneu, 2003.

9. Almeida LMC, Filho APN. Análise das cápsulas manipuladas segundo a RDC 67/2007 da ANVISA/MS para a garantia da qualidade. Rev. Bras. Farm., 91(3):119-25, 2010.

10. Brasil. Ministério da Saúde. Agência Nacional de Vigilância Sanitária. RDC n 67, de 08 de outubro de 2007. Dispõe sobre Boas Práticas de Manipulação de Preparações Magistrais e Oficinais para Uso Humano em farmácias. Brasília: Ministério da Saúde; 2007.

11. Nogueira LCL. Gerenciando pela qualidade total na saúde. 4. ed. Belo Horizonte: Editora de Desenvolvimento Gerencial; 2003.

12. Silva SH. Controle da qualidade assistencial de enfermagem: implementação de um modelo [Tese]. São Paulo (SP): Escola de Enfermagem de Ribeirão Preto/USP; 1994.

13. Carpenito LJ. Diagnóstico de Enfermagem e Planejamento do Cuidado. Carpenito LJ. Diagnóstico de Enfermagem: aplicação à prática clínica. 8. ed. Porto Alegre: Artmed; 2002. p. 68-91.

14. Vargas JO, Almeida AMDP, Vieira JGV, Faria AF. Análise dos procedimentos operacionais padronizados em um centro de distribuição de uma indústria alimentícia. In: Encontro Mineiro de Engenharia de Produção (EMEPRO). 4. 2008. Ouro Preto, MG. Resumos. Ouro Preto: EMEPRO; 2008.

15. Nunes PHC, Pereira BMG, Nominato JCS, Albuquerque EM, Silva LFN, Castro IRS, et al. Intervenção farmacêutica e prevenção de eventos adversos. Rev Bras de Ciências Farmacêuticas. (44)4: 661-669,2008.

16. Ferracini FT, Almeida SM, Locatelli J, Petriccione S, Haga CS. Implantação e evolução da farmácia clínica no uso racional de medicamentos em hospital terciário de grande porte. Hospital Albert Einstein. São Paulo. (9)4:456-60, 2011.

17. Malik AM, Schiesari LMC. Qualidade na gestão local de serviços e ações de saúde. v. 3. São Paulo: Faculdade de Saúde Pública da USP; 1998.

18. Kotler P. Administração de marketing: análise, planejamento, implementação e controle. 5a ed. São Paulo: Atlas; 1998. 
19. Rossi CAV, Slongo LA. Pesquisa de satisfação de clientes: o estado-da-arte e proposição de um método brasileiro. Rev Adm Contemp 1998;2(1):10125.

Recebido: 26 de fevereiro de 2018. Publicado: xx

Correspondência: Ana Carolina Reis Guimarães. E-mail:

anacarolfarm@yahoo.com.br

Conflito de Interesses: os autores declaram não haver conflito de interesses.

(C) This is an Open Access article distributed under the terms of the Creative Commons Attribution License, which permits unrestricted use, distribution, and reproduction in any medium, provided the original work is properly cited 